\title{
In vivo endothelialization and neointimal hyperplasia assessment after rabbit carotid endarterectomy with bovine pericardium
}

\author{
Yanfei Chen ${ }^{1,2 \#}$, Yao Feng ${ }^{1,2 \#}$, Tao Wang ${ }^{1,2 \#}$, Xiao Zhang ${ }^{1,2}$, Meng Zhang ${ }^{3}$, Xuesong Bai ${ }^{1,2}$, Long Li $^{1,2}$, \\ Kun Yang ${ }^{4}$, Yan $\mathrm{Ma}^{1,2}$, Zhiping Zhang ${ }^{1,2}$, Liqun Jiao ${ }^{1,2,5}$ \\ ${ }^{1}$ Department of Neurosurgery, Xuanwu Hospital, Capital Medical University, Beijing, China; ${ }^{2}$ China International Neuroscience Institute (China- \\ INI), Beijing, China; ${ }^{3}$ Department of Pathology, Xuanwu Hospital, Capital Medical University, Beijing, China; ${ }^{4}$ Department of Evidence-Based \\ Medicine, Xuanwu Hospital, Capital Medical University, Beijing, China; ${ }^{5}$ Department of Interventional Neuroradiology, Xuanwu Hospital, Capital \\ Medical University, Beijing, China \\ Contributions: (I) Conception and design: Y Chen, Y Feng, T Wang, L Jiao, Z Zhang; (II) Administrative support: None; (III) Provision of \\ study materials or patients: None; (IV) Collection and assembly of data: Y Chen, Y Feng, X Zhang, M Zhang, Z Zhang; (V) Data analysis and \\ interpretation: Y Chen, Y Feng, M Zhang, X Bai, Y Ma, Z Zhang, L Jiao, K Yang, T Wang; (VI) Manuscript writing: All authors; (VII) Final \\ approval of manuscript: All authors. \\ \#These authors contributed equally to this work. \\ Correspondence to: Liqun Jiao. Department of Neurosurgery and Department of Interventional Neuroradiology, Xuanwu Hospital, Capital Medical \\ University; China International Neuroscience Institute (China-INI), No. 45 Changchun Street, Xicheng District, Beijing 100053, China. \\ Email: liqunjiao@sina.cn; Zhiping Zhang. Department of Neurosurgery, Xuanwu Hospital, Capital Medical University; China International \\ Neuroscience Institute (China-INI), No. 45 Changchun Street, Xicheng District, Beijing 100053, China. Email: microsurgery_123@sina.com.
}

Background: Previous studies have reported that the use of a patch in carotid endarterectomy (CEA) surgery can reduce the rate of restenosis and perioperative complications. The goal of this study was to compare the short- and medium-term outcomes of endothelialization and neointimal hyperplasia of patch closure (PC) angioplasty in CEA with direct closure (DC) in a rabbit model. A bovine pericardial patch (BPP) was used in the PC procedures.

Methods: Two carotid arteries were dried by air flow to simulate endarterectomy and selected for PC and DC in each rabbit. Different animals were sacrificed at 1, 2, 3, 4, and 8 weeks after the procedure. The endarterectomized segments were extracted and examined microscopically with histopathological and immunohistochemical analysis, and electron-microscopy measurements.

Results: In all, 19 rabbits were included in this study; 3 rabbits were placed in a 2-week postoperative group and 4 rabbits were placed in the 1-, 3-, 4-, and 8-week postoperative group respectively. Hematoxylineosin (HE) staining showed neointima on the PC side at an early stage (1-week postoperatively), and intimal hyperplasia could be seen on both sides. Immunohistochemical analysis showed that Ki-67 was higher on the PC side than on the DC side at an early stage $\left(1,661.5 \pm 1,122.9\right.$ cells $\left./ \mathrm{mm}^{2}, \mathrm{P}=0.060\right)$. In the 2 -week postoperative group, von Willebrand factor (vWF) was higher on the DC side $\left(-377.0 \pm 155.6 \mathrm{cells} / \mathrm{mm}^{2}\right.$, $\mathrm{P}=0.052)$. Alpha-smooth muscle actin $(\alpha$-SMA) values were comparable on both sides $(\mathrm{P}>0.05)$. Electron microscopy measurements showed that functional endothelial cells exhibited a cobblestone-like morphology and were nicely elongated in the direction of blood flow.

Conclusions: The use of BPP in PC angioplasty during CEA can maintain stability and also provide rapid endothelialization. PC with BPP has comparable ability of efficient endothelialization with DC, but is more likely to have early endothelialization.

Keywords: Atherosclerosis; patch; neointima; carotid endarterectomy (CEA); animal model

Submitted Dec 21, 2020. Accepted for publication Feb 24, 2021.

doi: 10.21037/atm-20-8103

View this article at: http://dx.doi.org/10.21037/atm-20-8103 


\section{Introduction}

Carotid endarterectomy (CEA) is a durable procedure to prevent neurological symptoms and stroke in patients with significant internal carotid artery stenosis (1), even in elderly patients (2). Some randomized controlled trials have demonstrated the safety and efficacy of CEA $(3,4)$. Several authorities have shown the reduced incidence of both early restenosis and acute perioperative occlusion in patients treated with patch angioplasty compared with those treated with direct closure (DC) after CEA (5-7). According to the 2011 American Stroke Association guidelines, CEA with patch closure (PC) reduces the risk of occlusion, restenosis, and combined stroke or death (8). The current European Society for Vascular Surgery guidelines on carotid artery disease recommend routine PC after CEA (9).

A variety of patch materials are available for closure of the arteriotomy, including autologous venous biomaterial patches (which are derived from animal tissues) and synthetic patches such as expanded polytetrafluoroethylene (PTFE) and Dacron. Among them, the bovine pericardial patch (BPP) $(10,11)$ is the most popular option. BPP in human carotid artery repair was first introduced by Biasi et al. in 1996 (12). Since then, BPP has been commonly used in CEA procedures (1,13-15).

Although BPP has been available for more than 20 years, few studies have examined neointimal hyperplasia in PC compared with DC in CEA. Ito et al. (16) carried out an autopsy on a carotid artery specimen in the acute stages after CEA with patch angioplasty. They suggested that the intrinsic arterial wall may have an important role in intimal regeneration after CEA; however, the neointima on the patch was not demonstrated due to insufficient implantation time. Previous studies have also explored the healing response after carotid arterial injury in balloon-injured carotid artery and PC for carotid arteriotomy (17-19). Yet, these studies did not clarify the difference between PC and DC after CEA. In addition, re-endothelialization on the patch has always been particularly concerning, as it is directly related to thrombosis (20). Moreover, it is also unclear whether the neointima on the patch is functional. The goal of this study was therefore to compare the shortand medium-term outcomes of endothelialization and neointimal hyperplasia of BPP angioplasty during CEA with DC in a rabbit model. We present the following article in accordance with the ARRIVE reporting checklist (available at http://dx.doi.org/10.21037/atm-20-8103).

\section{Methods}

\section{Animals}

Male New Zealand white rabbits weighing 2-3 kg were purchased from Beijing Regal Experimental Animal Breeding Center, Beijing, China (certificate no. SCXK 2019-0015/2019-0043). Experiments were performed under a project license (No.: 20181001) granted by institutional ethics committee of Xuanwu Hospital, Capital Medical University, in compliance with the National Institutes of Health Guide for the care and use of animals.

\section{BPP}

The patch (4X, Beijing Balance Medical Technology Co., Ltd., China) used for PC after CEA was made of bovine pericardium and was treated by a series of biochemical processes, including decellularization, immunogenicity removal, and cross linking (for extra strength). After being sterilized, the patch was stored in normal saline for bacteriostasis. The BPP, which is primarily used for cardiovascular reconstruction and repair, has already been granted marketing production approval by the Chinese Food and Drug Administration (CFDA). With patent treatment technology, BPP can reduce short-term thrombosis and long-term restenosis caused by membrane hyperplasia, and also improve the long-term patency rate of blood vessels after surgery. Its effectiveness has been verified through congenital heart disease operations after years of implantation.

\section{Management of the carotid artery}

The rabbits were anaesthetized using $1 \mathrm{~mL} / \mathrm{kg}$ of $2 \%$ phenobarbital sodium via intravenous bolus through the ear vein. Next, the dose was increased according to the awake state of the rabbits. A middle incision approximately $2 \mathrm{~cm}$ in length was made to the rabbits' neck to expose the carotid arteries. A mini aneurysm clip (FT7247, Aesculap AG, Braun, Germany) was used to block the proximal and distal ends of the carotid artery. The endothelium of the arterial lumen was then air dried for 10 minutes at $200 \mathrm{~mL} / \mathrm{min}$ via needle puncture to simulate the CEA procedure. The airflow rate was controlled using a glass rotameter (LZB-3, 30-300 mL/min, Senlod, China). This model has been described in previous literature $(21,22)$, with reports 

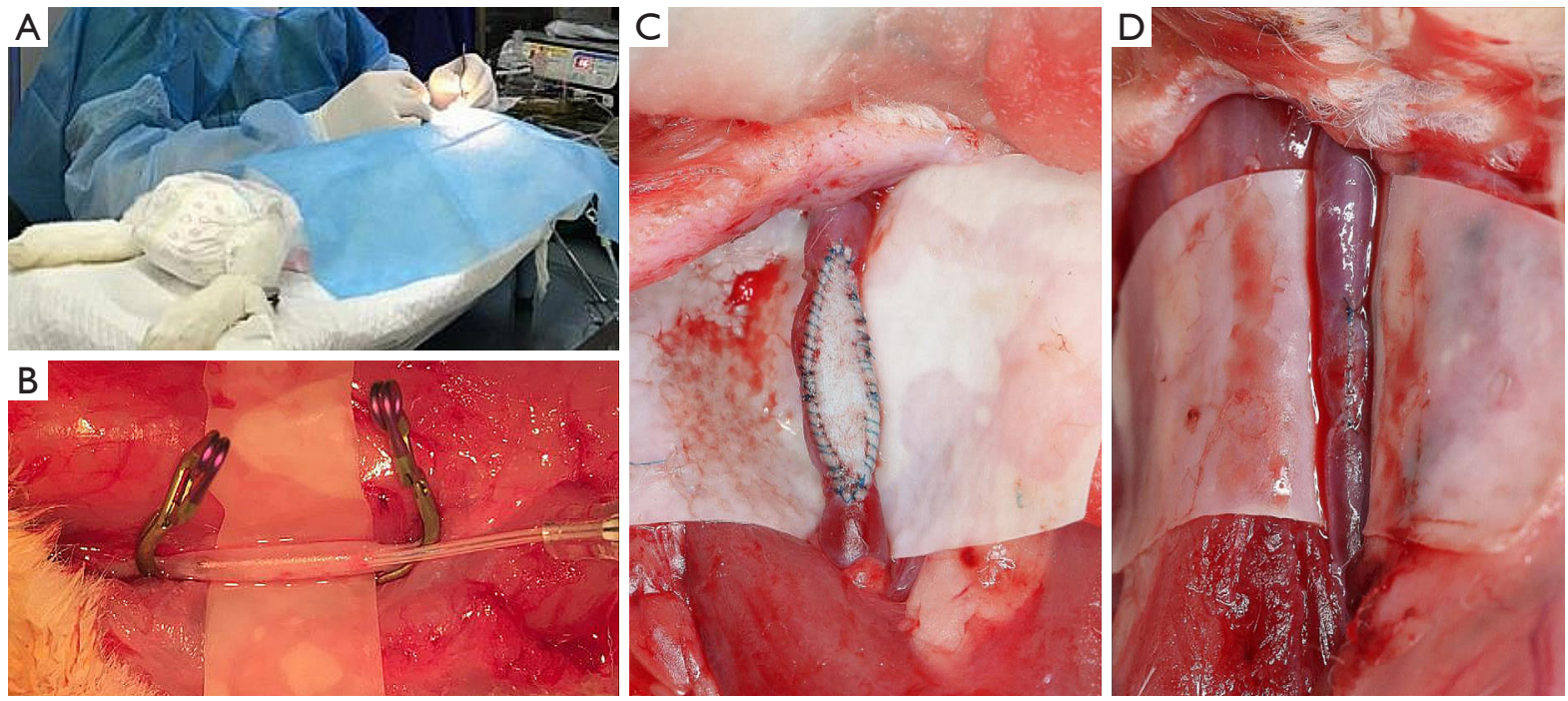

Figure 1 Operative situs. (A) Our surgical team member performing the surgery; (B) endothelial cells being damaged by air drying in order to simulate the carotid endarterectomy (CEA) procedure; (C) patch closure for the right carotid artery; (D) direct closure for the left carotid artery.

indicating that after 3 days, all the endothelial cells are removed from the full circumference of the segment of the artery exposed to air drying (23). The carotid artery was subsequently cut longitudinally. BPP closure was performed for the right carotid artery, while DC was performed for the left carotid artery (Figure 1). The operation was completed by suturing the incision, and the rabbits were allowed to wake up naturally from anesthesia. Following completion of the surgery, all experimental rabbits were transferred to the professional feeding center and fed a high-cholesterol diet.

\section{Experimental grouping}

We adopted the self-control method for comparison, performing CEA with PC in one carotid artery and DC in the other. All tested animals were divided into five groups based on different postoperative feeding and sacrificing times. The time intervals included 1, 2, 3, 4, and 8 weeks after the procedure. At each of these time points, the rabbits in the group were sacrificed, and their bilateral carotid arteries were removed for histopathological, immunohistochemical, and electron microscopy analysis.

\section{Histopathological and immunohistochemical analysis}

Each carotid artery specimen was divided into 2 parts from the middle line. One half was fixed in $10 \%$ neutral-buffered formalin, dehydrated in alcohol, and embedded in paraffin, and 2-4 $\mathrm{mm}$ transverse sections were made in the middle area of the graft or DC area. Samples were sectioned at a thickness of $5 \mu \mathrm{m}$ for immunohistochemical analysis and hematoxylin-eosin (HE) staining. Neointimal thickness was measured in HE-stained transverse sections which had been scanned into electronic documents. Neointimal thickness was measured from the middle area of the patch at 3 standardized positions using ImageScope (version 12.1.0, Aperio Technologies, Inc., USA). For DC, intimamedia thickness was measured from the area contrary to the suture line. Three points were also selected to calculate the average

Immunohistochemical analysis included monoclonal antibodies against alpha-smooth muscle actin ( $\alpha$-SMA) (mouse monoclonal antibody, dilution 1:200, GB23301, Servicebio, China) and von Willebrand factor (vWF) (rabbit monoclonal antibody, dilution 1:200, GB13030-2, Servicebio, China) to evaluate the genesis of the neointima. Monoclonal antibodies against Ki-67 (rabbit monoclonal antibody, dilution 1:200, GB23303, Servicebio, China), a nuclear protein associated with cellular proliferation, were used to investigate the proliferative activity of the neointima. For the DC side, the neointima was indistinguishable from the original structure, so we chose an area similar to the PC side for analysis. These immunohistochemical results were scanned (Pannoramic 250, 3D HISTECH Ltd., Hungary) 


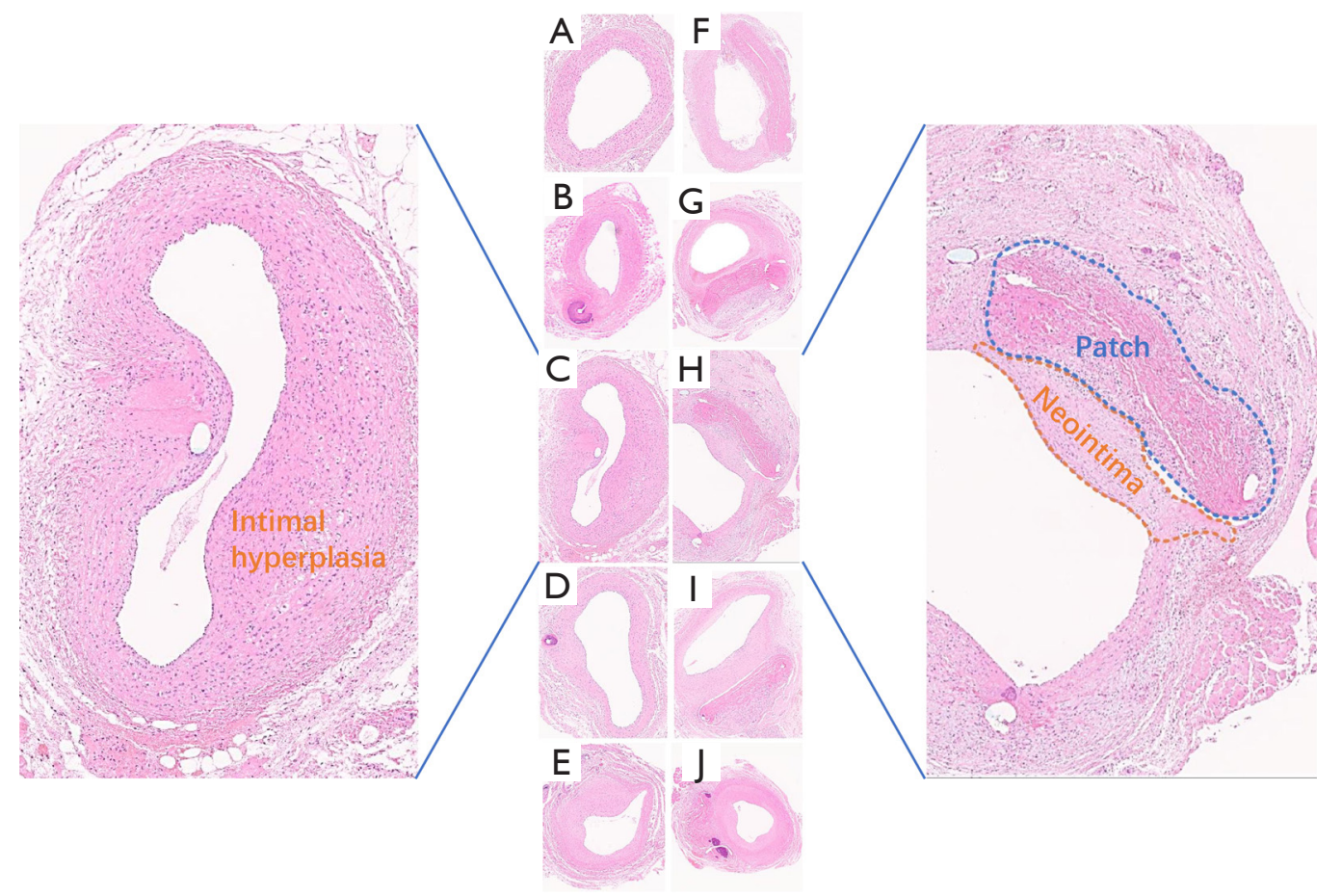

Figure 2 Hematoxylin-eosin staining of transverse sections through the segment of the vessels of patch closure and direct closure (A,B,C,D,E). The different postoperative feeding times for the direct closure side $(1,2,3,4$, and 8 weeks after surgery, respectively). Similarly, (F,G,H,I,J) show the different postoperative feeding times for the patch closure side, respectively. (C) and (H) are magnified, with clear intimal hyperplasia visible on $(\mathrm{C})$. On $(\mathrm{H})$, the patch is clearly differentiated from the intrinsic tissue, and neointima on the surface of the patch is obvious (A-J power of magnification: $\times 20$ ).

and analyzed quantitatively (HALO, Indica Labs, USA). Values in the results were the averages of counts from the indicated amounts of animals.

\section{Electron microscopy measurements}

Scanning electron microscopy (SEM) (SU8100, Hitachi, USA) and transmission electron microscopy (HT7800, Hitachi, USA) were used in the 5 groups. This permitted a better comparison of morphological aspects of the regenerated endothelium and the histological characteristics of the cells. Endothelial cell counts on SEM were analyzed by Image-pro plus 6.0 (Media Cybernetics, Inc., USA).

\section{Statistical analysis}

Continuous variables are expressed as mean values with standard deviation (SD), while categorical data are expressed as percentages. Comparisons between the PC and DC groups were performed using the Student's $t$-test or
Mann-Whitney $\mathrm{U}$ test for continuous variables. The level of significance was defined as a $\mathrm{P}$ value $<0.05$.

\section{Results}

A total of 25 rabbits were included in this study. Five rabbits died during the perioperative period, which included 2 deaths due to anesthesia overdose and 3 deaths due to the surgical procedure. The other rabbit died during the feeding period in the 2 -week postoperative group. Consequently, 19 rabbits were included in our analysis, with just 3 rabbits in the 2-week postoperative group and 4 rabbits in each of the other groups. A flow chart of experimental rabbits is shown in Figure S1.

When the specific feeding time arrived, we harvested the rabbits' carotid arteries. Figure 2 shows the HEstained sections of the PC and DC sides. As time elapsed, intimal hyperplasia occurred on both sides. The highcholesterol diet accelerated the intimal hyperplasia of the rabbits' carotid arteries. At an early stage (1 week 

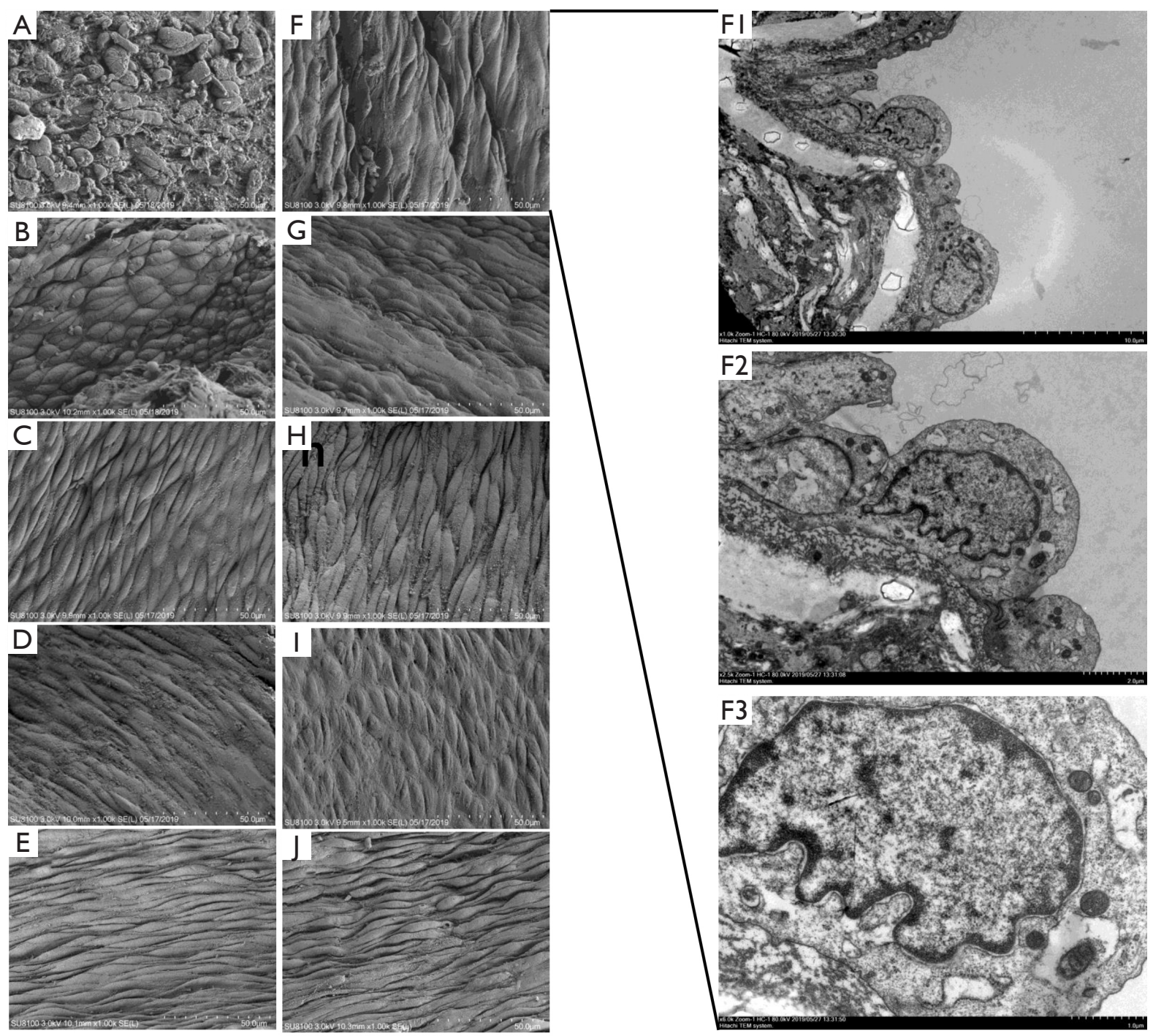

Figure 3 Representative scanning electron microphotographs and transmission electron microphotographs of the inner surface of the patch or vessel (A,B,C,D,E). The different postoperative feeding times for the direct closure side $(1,2,3,4$, and 8 weeks after surgery, respectively). Similarly, (F,G,H,I,J) show the different postoperative feeding times the for patch closure side, respectively. (F1, F2, F3) The microstructure of endothelial cells on the patch closure side 1 week after surgery. Endothelial cells have a complete variety of organelles that are normal in shape [power of magnification for $(A, B, C, D, E, F, G, H, I, J): \times 1,000$; power of magnification for F1: $\times 1,000$; power of magnification for F2: $\times 2,500$; power of magnification for $\mathrm{F} 3: \times 6,000]$.

postoperatively), neointima was observed on the inner surface of the patch, and the thickness increased over time. Subsequently, scanning electron microphotographs showed that endothelialization occurred in the first week on the patch side. Endothelial cells exhibited a cobblestone-like morphology and were nicely elongated in the direction of blood flow, which was similar to the normal morphologic features of the endothelium in the DC side. Also, compared to DC side, endothelialization occurred earlier on the PC side. Transmission electron microphotographs showed that the endothelial cells had a complete variety of organelles that were normal in shape (Figure 3). Moreover, we compared the immunohistochemical results between the PC and DC sides (Table 1). Both groups had different $\alpha$-SMA, vWF, and $\mathrm{Ki}-67$ values in the variable feeding times. In the early stages, Ki-67 was higher in 
Table 1 Differences between the PC and DC sides in immunohistochemistry over time

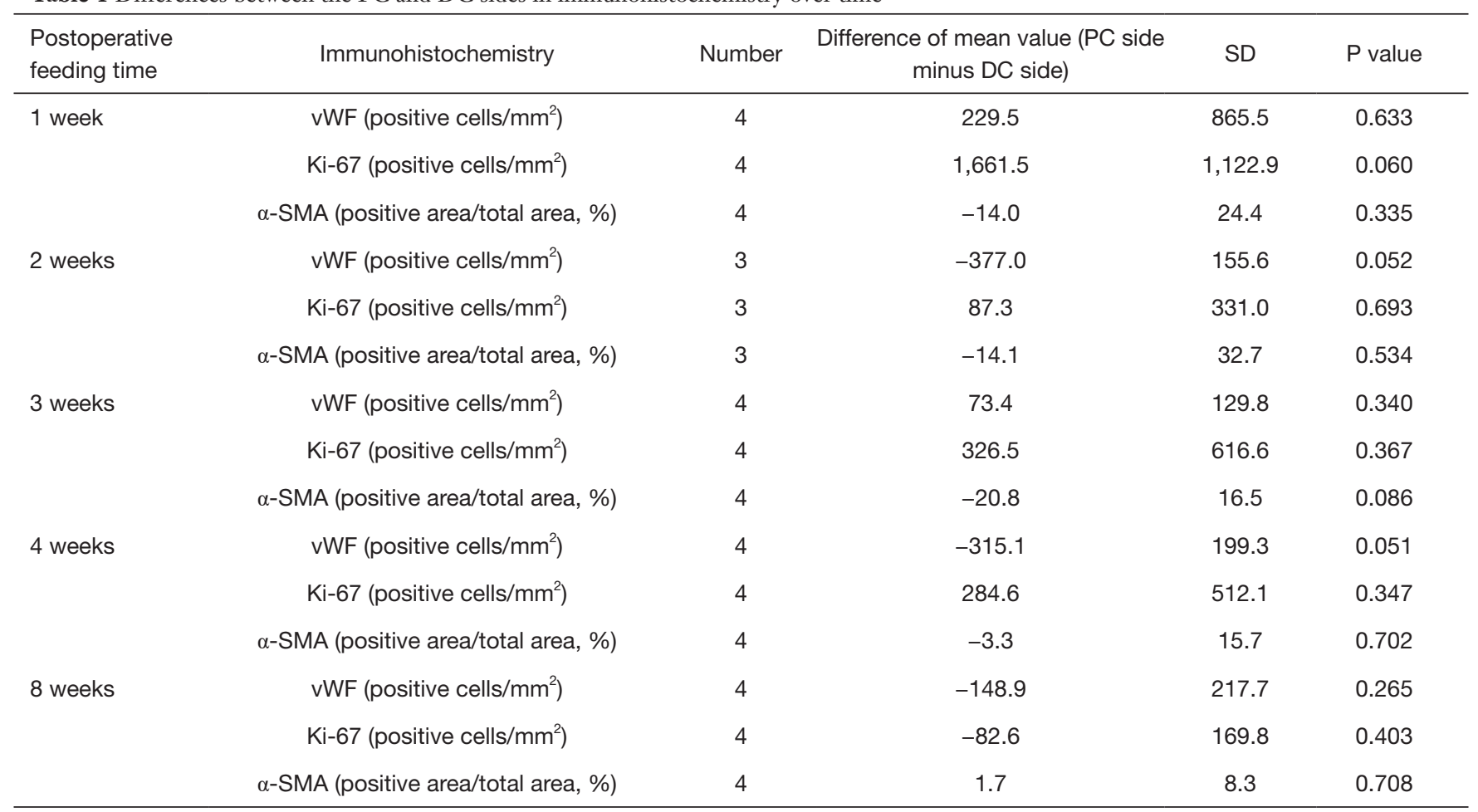

PC, patch closure; DC, direct closure; vWF, von Willebrand factor; $\alpha$-SMA, alpha-smooth muscle actin; SD, standard deviation.

the PC side $\left(1,661.5 \pm 1,122.9\right.$ cells $\left./ \mathrm{mm}^{2}, \mathrm{P}=0.060\right)$. At 2 weeks postoperatively, vWF was higher in the DC side $\left(-377.0 \pm 155.6\right.$ cells $\left./ \mathrm{mm}^{2}, \mathrm{P}=0.052\right)$. $\alpha$-SMA values were comparable for both sides $(\mathrm{P}>0.05)$. Although these differences did not reach statistical difference, they did reveal the potential mechanism for endothelialization in CEA with or without patch use.

As the feeding time increased, the thickness of the neointima on the PC side also increased. The thickness of the intimal media contrary to the suture line was relatively stable. The endothelial cell count was comparable in both the PC and DC sides; however, endothelial cells could appear earlier on the PC side (Figure 4).

\section{Discussion}

CEA is a proven treatment for extracranial carotid artery stenosis $(4,24)$. However, post-CEA intimal hyperplasia can complicate and reduce the effectiveness of endarterectomy. Some researchers discovered that expanding the lumen with a patch can help to reduce the risk of restenosis (1). A subgroup analysis of the CREST (Carotid Revascularization Endarterectomy Versus Stenting Trial) study showed that the use of a patch in CEA could significantly reduce the occurrence of restenosis after 2 years compared with DC ( $3.1 \%$ vs. $10.7 \%$, hazard ratio $=0.35)$ (5). At present, the use of patches may present some challenges, such as prolonged operation time, graft-related infection, thrombosis, aneurysmal dilatation, and other issues $(11,25)$. However, few studies have compared the intimal growth between PC and DC after CEA.

In the present study, we explored the difference in neointima between PC and DC groups via simulation of the CEA procedure in a rabbit model. The rabbits were fed a high-cholesterol diet after the procedure to simulate patients with poorly controlled risk factors, as a highcholesterol diet can accelerate the process of intimal hyperplasia (26). The results of HE staining showed that the BPP could be clearly distinguished from the surrounding vessel wall, indicating that this domestic BPP was very stable in the human body. As the feeding time increased, the proliferation of the intima became increasingly prominent. For the PC side, the intimal thickness grew fastest in the third week postoperatively. SEM results showed that the endothelial cells were relatively intact on the surface of the intima in the second week after surgery. The regenerated 


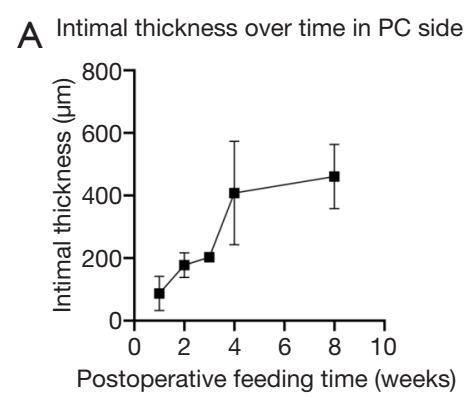

C Endothelial cell count in PC side

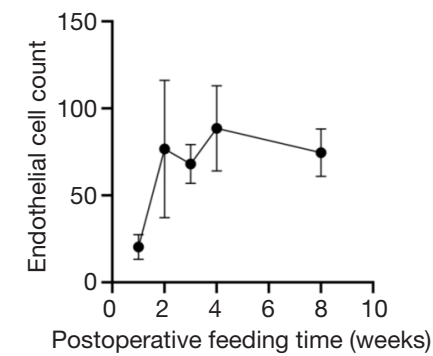

B

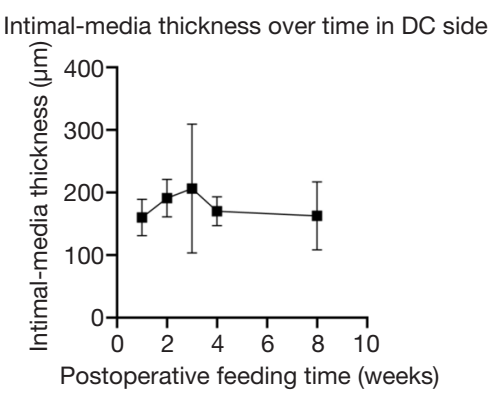

D

Endothelial cell count in DC side

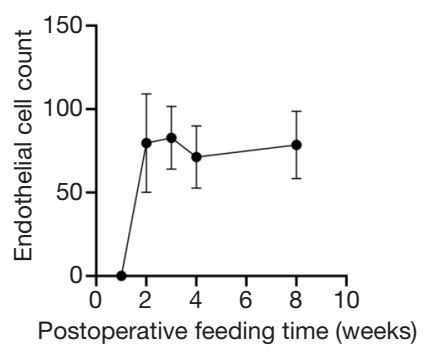

Figure 4 Quantitative analysis of the histopathological and scanning electron microphotographs findings. (A) Intimal thickness over time in the PC side; (B) intimal-media thickness over time in the DC side; (C) endothelial cell count in the PC side via scanning electron microphotography; (D) endothelial cell count in the DC side over time via scanning electron microphotograph. PC, patch closure; DC, direct closure.

endothelium showed normal anatomical characteristics, and its growth in the endarterectomized area was faster compared to the DC side. Thyberg et al. (27) also reported that the neointima of the carotid artery had almost reached its final size on the 14th day following balloon injury in a rat model. They found that these neointimal cells maintained a synthetic phenotype with prominent secretary organelles. Our findings are consistent with these results. The endothelial cells had a complete variety of organelles that were normal in shape, indicating that the neointimal cells on the PC side were functional.

Through immunohistochemical examination of the carotid arteries of rabbits with different feeding times, we found that $\mathrm{Ki}-67$ was higher on the PC side in the first feeding week and $\mathrm{vWF}$ was generally higher on the DC side. We hypothesized that the domestic BPP might stimulate cell proliferation, which could be beneficial for re-endothelialization. In fact, recruitment of circulating endothelial progenitor cells plays an important role in vascular repair re-endothelialization (28), which might explain this phenomenon in the present study. The fact that $\mathrm{vWF}$ was higher on the DC side may be due to the neointima on the DC side being more derived from endothelial cells as compared to the PC side. There was no difference in the $\alpha$-SMA value between both sides. We speculated that the likely reason for this is that most of the neointima was still derived from smooth muscle cells.

It is understood that thrombosis often occurs in a region of endothelial cell injury and that early endothelialization can effectively prevent thrombosis (29). The endothelialization ability observed in our study was equivalent between the PC and DC sides, although the PC side tended to re-endothelialize earlier. This feature could likely reduce complications or shorten the postoperative usage time of dual-antiplatelet drugs.

The use of patch suture after CEA has been demonstrated to effectively reduce the incidence of restenosis 2 years after operation ( $3.1 \%$ vs. $10.7 \%, \mathrm{P}<0.0001)$, and the incidence of events at the end of the perioperative period and 4-year follow-up has been found to be lower than that of direct suture (5). Bond et al. conducted a systematic review of randomized controlled studies comparing carotid artery patch suture with direct suture or different patch materials. The results showed that the use of patch could effectively reduce the occurrence of stroke during the perioperative period and the occurrence of stroke and restenosis during long-term follow-up (30). Therefore, the development of the patch can benefit clinical practice, and this new type 
of patch is also expected to play a certain role in future vascular surgery.

This study had several limitations that should be noted. First, we did not describe the percentage of endothelium covering the intima, but indirectly reflected this feature through SEM. Secondly, we did not describe the restenosis of the lumen. We believed that the thickness of intimal hyperplasia could also indirectly reflect restenosis. Thirdly, this study did not investigate the related mechanisms (e.g., protein, signaling pathways), and we aim to further explore this area in the future. Fourthly, the sample size of this study was small, and thus, we recommend a cautious interpretation of the results.

\section{Conclusions}

Applying BPP in CEA can maintain stability in a rabbit model and facilitate rapid endothelialization. PC with BPP has a comparable ability of efficient endothelialization with DC; however it is more likely to produce early endothelialization. The results of this research suggest promise in the use of BPP in clinical practice although the specific mechanisms involved still need to be further investigated using a larger sample size.

\section{Acknowledgments}

Funding: This study was funded by the National Key Research and Development Project (No. 2016YFC1301703).

\section{Footnote}

Reporting Checklist: The authors have completed the ARRIVE reporting checklist. Available at http://dx.doi. org/10.21037/atm-20-8103

Data Sharing Statement: Available at http://dx.doi. org/10.21037/atm-20-8103

Conflicts of Interest: All authors have completed the ICMJE uniform disclosure form (available at http://dx.doi. org/10.21037/atm-20-8103). The authors have no conflicts of interest to declare.

Ethical Statement: The authors are accountable for all aspects of the work in ensuring that questions related to the accuracy or integrity of any part of the work are appropriately investigated and resolved. Experiments were performed under a project license (No.: 20181001) granted by institutional ethics committee of Xuanwu Hospital, Capital Medical University, in compliance with the National Institutes of Health Guide for the care and use of animals.

Open Access Statement: This is an Open Access article distributed in accordance with the Creative Commons Attribution-NonCommercial-NoDerivs 4.0 International License (CC BY-NC-ND 4.0), which permits the noncommercial replication and distribution of the article with the strict proviso that no changes or edits are made and the original work is properly cited (including links to both the formal publication through the relevant DOI and the license). See: https://creativecommons.org/licenses/by-nc-nd/4.0/.

\section{References}

1. Oldenburg WA, Almerey T, Selim M, et al. Durability of Carotid Endarterectomy with Bovine Pericardial Patch. Ann Vasc Surg 2018;50:218-24.

2. Feng Y, Bai X, Wang T, et al. Thirty-day outcomes of carotid endarterectomy in the elderly: A 17-year singlecenter study. J Clin Neurosci 2020;78:86-90.

3. Barnett HJ, Taylor DW, Eliasziw M, et al. Benefit of carotid endarterectomy in patients with symptomatic moderate or severe stenosis. North American Symptomatic Carotid Endarterectomy Trial Collaborators. N Engl J Med 1998;339:1415-25.

4. Endarterectomy for asymptomatic carotid artery stenosis. Executive Committee for the Asymptomatic Carotid Atherosclerosis Study. JAMA 1995;273:1421-8.

5. Malas M, Glebova NO, Hughes SE, et al. Effect of patching on reducing restenosis in the carotid revascularization endarterectomy versus stenting trial. Stroke 2015;46:757-61.

6. Loftus CM. Technical aspects of carotid endarterectomy with Hemashield patch graft. Neurol Med Chir (Tokyo) 1997;37:805-18.

7. Clagett GP, Patterson CB, Fisher DF Jr, et al. Vein patch versus primary closure for carotid endarterectomy. A randomized prospective study in a selected group of patients. J Vasc Surg 1989;9:213-23.

8. Brott TG, Halperin JL, Abbara S, et al. 2011 ASA/ACCF/ AHA/AANN/AANS/ACR/ASNR/CNS/SAIP/SCAI/SIR/ SNIS/SVM/SVS guideline on the management of patients with extracranial carotid and vertebral artery disease: executive summary. Stroke 2011;42:e420-63.

9. Naylor AR, Ricco JB, de Borst GJ, et al. Editor's Choice 
- Management of Atherosclerotic Carotid and Vertebral Artery Disease: 2017 Clinical Practice Guidelines of the European Society for Vascular Surgery (ESVS). Eur J Vasc Endovasc Surg 2018;55:3-81.

10. Texakalidis P, Giannopoulos S, Charisis N, et al. A meta-analysis of randomized trials comparing bovine pericardium and other patch materials for carotid endarterectomy. J Vasc Surg 2018;68:1241-56.e1.

11. Muto A, Nishibe T, Dardik H, et al. Patches for carotid artery endarterectomy: current materials and prospects. J Vasc Surg 2009;50:206-13.

12. Biasi GM, Mingazzini P, Baronio L, et al. Processed bovine pericardium as patch angioplasty for carotid endarterectomy: a preliminary report. Cardiovasc Surg 1996;4:591-5.

13. Dorweiler B, Kayser C, Zipp F, et al. Long-term performance of the bovine pericardium patch in conventional carotid endarterectomy. Thorac Cardiovasc Surg 2015;63:168-74.

14. Kim JH, Cho YP, Kwon TW, et al. Ten-year comparative analysis of bovine pericardium and autogenous vein for patch angioplasty in patients undergoing carotid endarterectomy. Ann Vasc Surg 2012;26:353-8.

15. Marien BJ, Raffetto JD, Seidman CS, et al. Bovine pericardium vs dacron for patch angioplasty after carotid endarterectomy: a prospective randomized study. Arch Surg 2002;137:785-8.

16. Ito $M$, Niiya $Y$, Uchino $H$, et al. Irregular neointimal lining with prominent proliferative activity after carotid patch angioplasty: an autopsy case report. World Neurosurg 2014;82:240.e1-6.

17. Liu WS, Lin PC, Chang LF, et al. Inhibitory effect of n-butylidenephthalide on neointimal hyperplasia in balloon injured rat carotid artery. Phytother Res 2011;25:1494-502.

18. Ao PY, Hawthorne WJ, Vicaretti M, et al. Development of intimal hyperplasia in six different vascular prostheses. Eur J Vasc Endovasc Surg 2000;20:241-9.

19. Rhee RY, Gloviczki P, Cambria RA, et al. Experimental evaluation of bleeding complications, thrombogenicity and neointimal characteristics of prosthetic patch materials used for carotid angioplasty. Cardiovasc Surg 1996;4:746-52.

20. Jalaie H, Steitz J, Afify $M$, et al. In vivo endothelialization and neointimal hyperplasia assessment after angioplasty of sheep carotid artery with a novel polycarbonate polyurethane patch. J Biomater Appl 2019;34:208-18.

21. Chen X, Ren S, Ma MG, et al. Hirulog-like peptide reduces restenosis and expression of tissue factor and transforming growth factor-beta in carotid artery of atherosclerotic rabbits. Atherosclerosis 2003;169:31-40.

22. Lafont A, Guzman LA, Whitlow PL, et al. Restenosis after experimental angioplasty. Intimal, medial, and adventitial changes associated with constrictive remodeling. Circ Res 1995;76:996-1002.

23. Richardson M, Hatton MW, Buchanan MR, et al. Wound healing in the media of the normolipemic rabbit carotid artery injured by air drying or by balloon catheter deendothelialization. Am J Pathol 1990;137:1453-65.

24. Barnett HJM, Taylor DW, Haynes RB, et al. Beneficial effect of carotid endarterectomy in symptomatic patients with high-grade carotid stenosis. N Engl J Med 1991;325:445-53.

25. Uno M, Takai H, Yagi K, et al. Surgical Technique for Carotid Endarterectomy: Current Methods and Problems. Neurol Med Chir (Tokyo) 2020;60:419-28.

26. Kisanuki A, Asada Y, Hatakeyama K, et al. Contribution of the endothelium to intimal thickening in normocholesterolemic and hypercholesterolemic rabbits. Arterioscler Thromb 1992;12:1198-205.

27. Thyberg J, Blomgren K, Hedin U, et al. Phenotypic modulation of smooth muscle cells during the formation of neointimal thickenings in the rat carotid artery after balloon injury: an electron-microscopic and stereological study. Cell Tissue Res 1995;281:421-33.

28. Fujiyama S, Amano K, Uehira K, et al. Bone marrow monocyte lineage cells adhere on injured endothelium in a monocyte chemoattractant protein-1-dependent manner and accelerate reendothelialization as endothelial progenitor cells. Circ Res 2003;93:980-9.

29. De Visscher G, Mesure L, Meuris B, et al. Improved endothelialization and reduced thrombosis by coating a synthetic vascular graft with fibronectin and stem cell homing factor SDF-1 $\alpha$. Acta Biomater 2012;8:1330-8.

30. Bond R, Rerkasem K, Naylor AR, et al. Systematic review of randomized controlled trials of patch angioplasty versus primary closure and different types of patch materials during carotid endarterectomy. J Vasc Surg 2004;40:1126-35.

Cite this article as: Chen Y, Feng Y, Wang T, Zhang X, Zhang M, Bai X, Li L, Yang K, Ma Y, Zhang Z, Jiao L. In vivo endothelialization and neointimal hyperplasia assessment after rabbit carotid endarterectomy with bovine pericardium. Ann Transl Med 2021;9(6):471. doi: 10.21037/atm-20-8103 


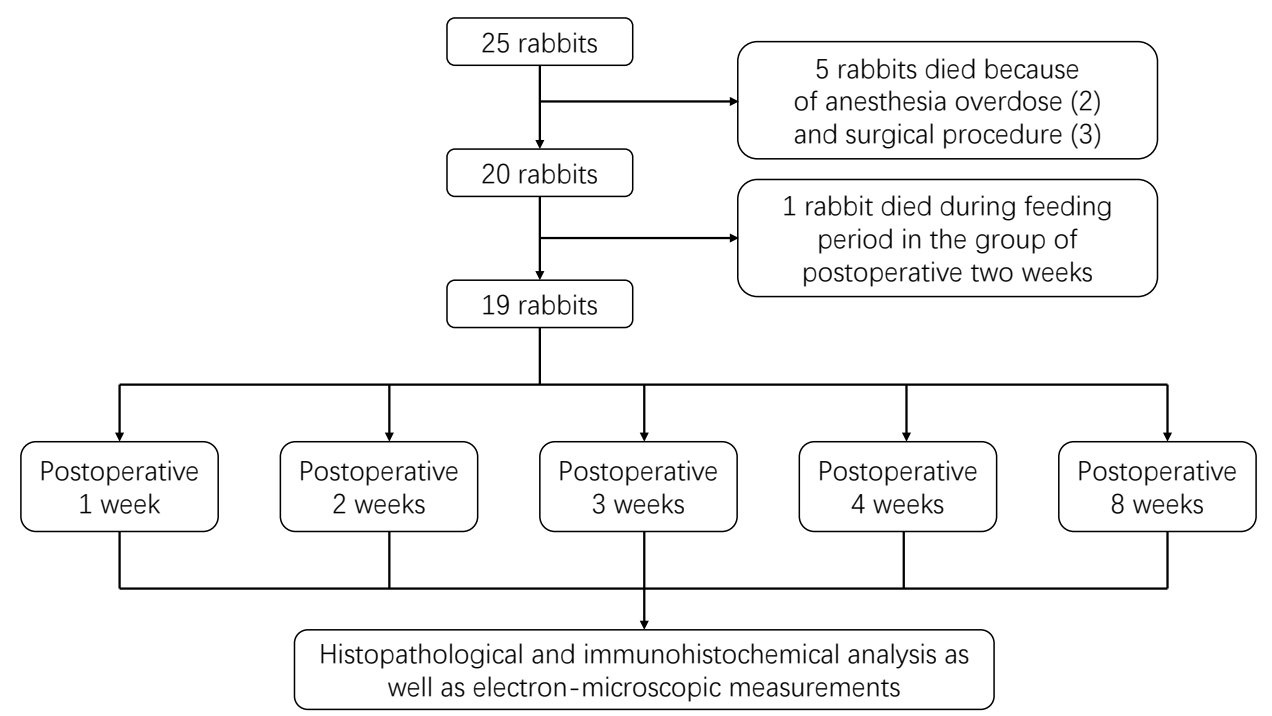

Figure S1 Flow chart of the experimental rabbit model. 